\title{
SIMULATING ORDER FULFILLMENT AND SUPPLY PLANNING FOR A VERTICALLY ALIGNED INDUSTRY SOLUTION BUSINESS
}

\author{
Feng Cheng \\ Young M. Lee \\ IBM T.J. Watson Research Center \\ 1101 Kitchawan Road \\ Yorktown Heights, New York 10598 \\ USA
}

\author{
Hong Wei Ding \\ Wei Wang \\ IBM China Research Lab \\ Zhong Guang Cun Software Park \\ Beijing, 100193 \\ CHINA
}

\author{
Stuart Stephens \\ IBM Integrated Supply Chain \\ 3039 Cornwallis Road \\ Raleigh NC 27709 \\ USA
}

\begin{abstract}
We model supply chain of an industry solution equipment manufacturer, where the merchandise is sold worldwide, but suppliers are mostly located in Asia. The preferred shipment of supply is the ocean shipment, and it takes about 5 weeks. Premium air shipment can be used to expedite delivery, but it costs substantially higher. To hedge against variability of demand and to satisfy customer service level, a certain level of safety stock is needed but inventory carrying cost can be high. Therefore, a careful fulfillment strategy is very important to balance serviceability and cost. A supply chain simulation model is developed to analyze the order fulfillment and supply planning process for the business to identify efficient supply chain strategy. The model simulates and evaluates three key performance metrics; serviceability, inventory costs and premium transportation costs, and their interactions.
\end{abstract}

\section{INTRODUCTION}

In a globally integrated and lean supply chain, distributors often rely on a single supplier, which can be located far away from the fulfillment locations experiencing long transportation lead time and high risk of customer service. In this work, we model supply chain of an industry solution manufacturer, where the merchandise is sold worldwide, but component suppliers are mostly located in Asia. The manufacturer provides systems for a wide range of customers from food service and hospitality businesses to grocery and general merchants including supermarkets and department stores. A complete system for a typical environment includes a system unit with 5-7 I/O devices. The supply chain under consideration consists of three tiers (see Figure 1). Tier 1 suppliers are fulfillment centers operating in each of the geographical regions to provide quick product delivery and service to customers. The configuration of the final products are carried out in the fulfillment centers based on customer orders. The components of the products are manufactured by Tier 2 suppliers usually in advance of customer orders based on the forecasted demand and shipped from Asia where most of the Tier 2 suppliers are located. In order to achieve the desired serviceability target, some level of safety stock inventory must be maintained at the fulfillment centers to protect against the demand uncertainty associated with the long supply and transportation lead time. Air shipments are sometimes used to improve the responsiveness to urgent customer orders. However, air shipments are used only when there is a justifiable business need since its cost is substantially higher. Therefore, the task of managing the inventory costs and transportation costs as well as the serviceability requirements becomes a complex and challenging supply chain optimization problem.

To efficiently and profitably fulfill the orders, an optimal decision that considers and balances the tradeoffs between various cost and serviceability factors need to be made. However, little has been reported in practice or literature for optimization problems with joint inventory replenishment decision and transportation decision. Rather, the fulfillment decision is typically made by simple ad-hoc, business rules. Recent research described in Sethi et al. (2001), Zhang (1996), and Scheller-Wolf et al. (1998), extended the standard model in the literature by allowing multiple delivery modes. However, the solution methods proposed in Sethi et al. and Zhang are based on periodic planning models with assumptions that the transportation lead times must be consecutive. The decision rules derived from the existing methods typically deal with stationary demand only. They do not take into account the different demand priorities of orders. Furthermore, inventory deci- 
sions in Scheller-Wolf et al. (1998) are made based on the inventory position only without analyzing the pipeline inventory information at each stage of the supply chain.

\section{SUPPLY CHAIN ENVIRONMENT}

To deal with this type of complex supply chain problem, we adopt a simulation-based solution approach which provides the flexibility for building realistic supply chain models. IBM Research has developed "best-of breed" supply chain simulation tools including Supply Chain Analyzer (Bagchi et al. 1998), which enjoyed considerable success both in the IBM internal supply chain and in external consulting. However, there is a need to build a next generation supply chain modeling tool incorporating the state of art software development technology and bringing in enhanced modeling and simulation capability to the new tool. IBM China Research Lab developed a new supply chain modeling tool SmartSCOR (Dong et al., 2006), and recently its supply chain simulation part of the tool became a standalone tool named GBSE. It is an integrated supply chain simulation tool to help making tactical level decisions. It supports industry-scale data sets and a rich set of supply chain processes. GBSE is based on Eclipse platform and implemented as an Eclipse RCP (Rich Client Platform).

For the purpose of this study, we further developed additional modeling features that are required for modeling the supply chain. An important feature of the enhanced GBSE is the complementary functionality of the optimization and simulation engines. With the optimization engine, the user can perform fast yet detailed what-if analyses, which are beyond the capability of most standard simulation tool. The GBSE simulation engine allows users to simulate various supply chain policies, and to evaluate the effectiveness of the supply plans generated by the optimization engine.

Figure 1 illustrates a typical supply chain system modeled by GBSE. The supply chain operation consists of two sub-processes: planning and execution. The planning process includes demand forecasting, which is based on historical order data and other considerations, and supply planning, which creates a supply commitment from components suppliers. The execution process starts at the order processing after orders are received from customers. The order fulfillment and scheduling module generates the purchase orders to the suppliers as well as the premium ship approvals which authorize the use of air shipments.

The simulation engine provides modeling functions for the following components of the supply chain:

- Customer represents external customers that issue orders to the supply chain, based on demand forecast. It can also model information about the desired service level and priority for the customer.

- Manufacturing models assembly process and keeps raw materials and finished goods inventory.

- Distribution models distribution centers, including finished goods inventory and material handling.

- Transportation models transportation time, vehicle loading, and transportation costs.

- Forecasting models demand forecast of products, including promotional and stochastic demand.

- Inventory planning models periodic setting of inventory target levels. Underlying this process is

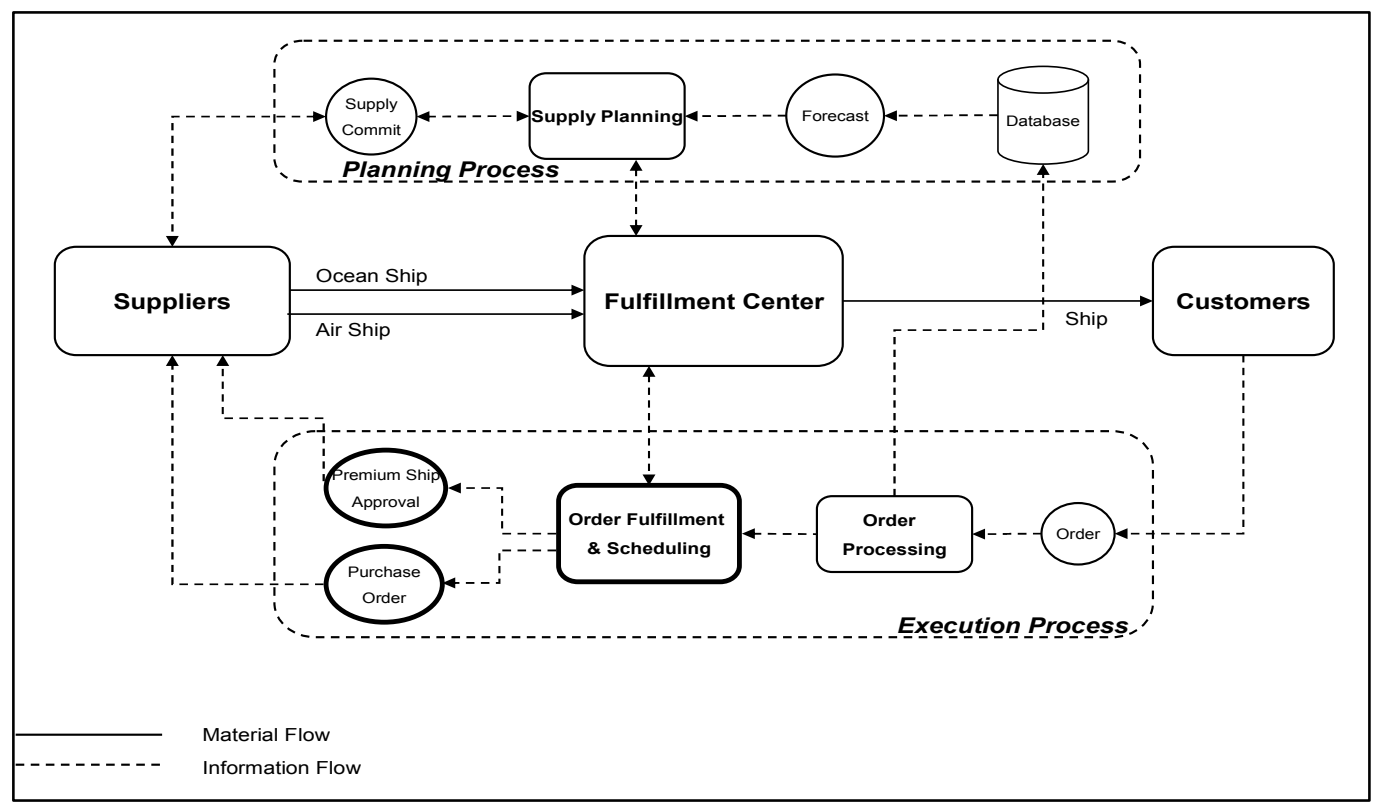

Figure 1. Functional Components of a Supply Chain Model 
the GSBE optimization engine that computes recommended inventory levels at various locations in the supply chain based on desired customer serviceability.

The simulation engine also contains an animation module that allows users to see how materials and information flow through the supply chain.

GBSE utilizes IBM's Supply Capability Engine (SCE) (Dietrich, et. al, 2005) to optimize the production planning problem based on the forecasted demand, which is an immensely challenging task in itself given that a supply chain typically involves hundreds to thousands of part numbers with multi-level bills-of-materials and associated lead times and costs, and many manufacturing and distribution sites linked by different transportation modes. In GBSE, SCE is invoked periodically during the simulation to recalculate target inventory levels according to the latest update of demand forecast. By combining the simulation and optimization, the tool provides the capability for performing quick, what-if analyses on different business scenarios that are beyond that of the conventional simulation model.

\section{SIMULATION MODEL}

The base model for the simulation study is derived from a real-world problem compiled from the actual data of the supply chain.

\subsection{Description of the Supply Chain Model}

As shown in Figure 2, we model the supply chain as a Three-Tier Supply Chain with several Tier 1 suppliers as fulfillment centers serving customers in Americas, Europe and Asia respectively. The fulfillment center in a geographic region performs the final system configurations based on customer orders and ship the products to the customers in the region. The final configuration is usually a quick process with relatively short processing times.

On the other hand, the supply lead times from the Tier 2 suppliers to Tier 1 suppliers (fulfillment centers) are usually 4-5 weeks long given that all of the tier 2 suppliers, which manufacture and supply the system units, are all located in Asia. Tier 3 suppliers are parts suppliers for various sub-components, and they are not modeled explicitly in the supply chain model. However, the supply lead times from Tier 3 suppliers are captured as part of Tier 2 suppliers' manufacturing lead times.

The product portfolio modeled has a wide ranges of product offering with several product families. In the simulation model, the Bill-of-Material (BOM) consists of two levels: products and components. For each product, the following are specified: the parent-child relationship, the usage rate of parts, and the assembly lead time required for building the product.

There are two types of forecasts used in the planning process. One is the demand forecast at the product level. The other is the feature ratio feature that determines the required quantities of the components for each product. The

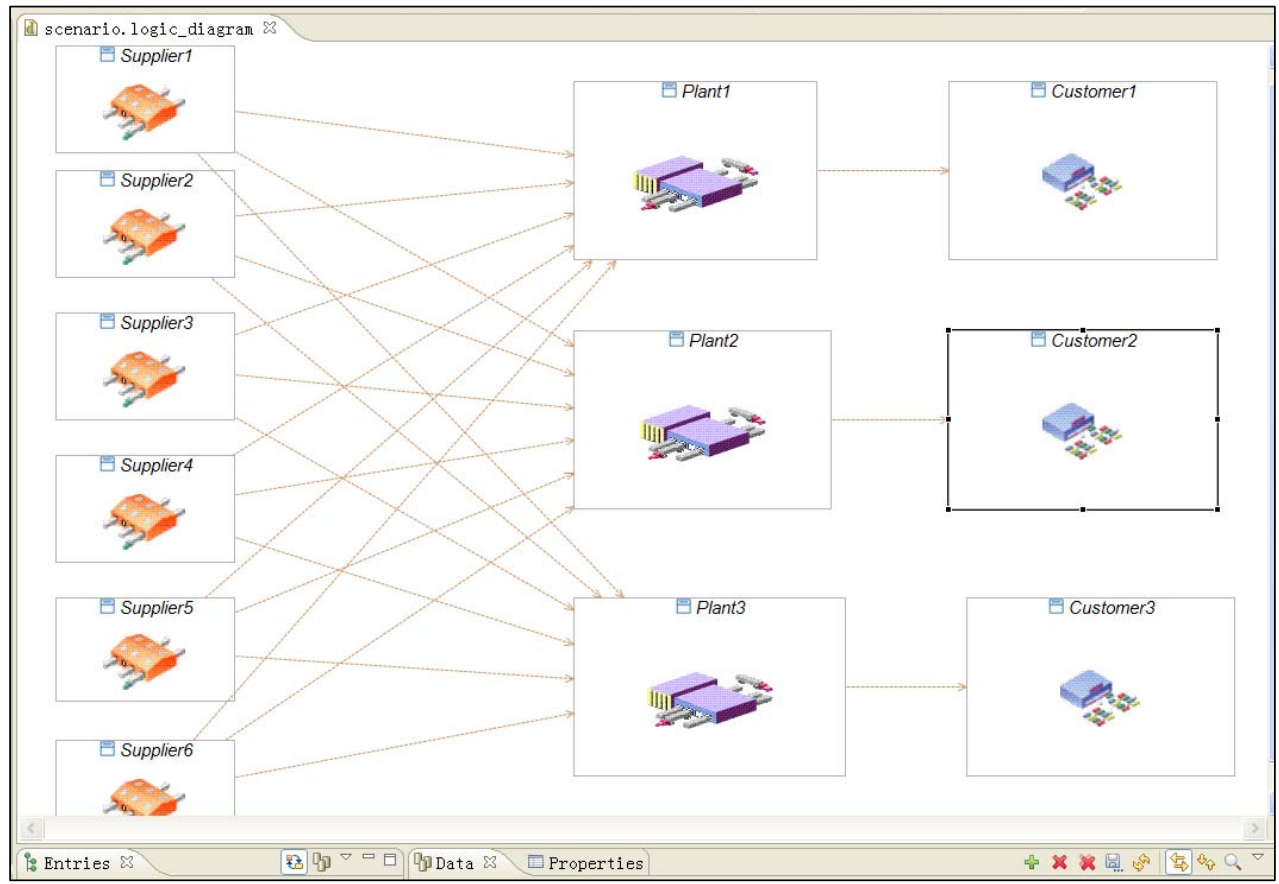

Figure 2. The Supply Chain Network 
feature ratios also reflect the customer specific requirements, e.g., the color of the system. The proliferation of features in fact increases the difficulty of forecasting demand significantly.

Given the long supply lead time and the complexity of product offerings, managing the parts inventory to meet the customer demand with a high service level is always a challenging task for the business. There are two types of inventory control strategies that have been used for each of the Tier 1 inventory stocking points, i.e., build to plan (BTP) and build to order (BTO). In most of the cases, BTP is used because the lead time needed for BTO would be unacceptable for fulfilling customer orders.

However, part of the long supply lead time is due to the ocean transportation used for shipments from Asia to the other regions, and it can be substantially reduced by switching to the air transportation method at a premium cost. The tradeoff between the supply lead times and shipping costs is no doubt a key issue to be addressed in the simulation study.

\subsection{Input Data for Simulation Model}

GBSE provides three convenient ways to manage the model data. A user can view and edit the data interactively using the graphical user interface of the tool. Excel spreadsheets can also be used to import or export the data in or out of the tool. Furthermore, a DB2 database is used to store all model data and simulation outputs. In a typical supply chain simulation, the following input parameters are specified:

- number and location of suppliers, manufacturers, and distribution centers

- inventory level of each product at each site

- manufacturing and replenishment policies: buildto-plan, build-to-order, etc.

- transportation policies: ocean shipment or air shipment determined based on order priority, promised ship date, and transportation budget constraint.

- manufacturing and assembly lead times

- demand variability

\subsection{Simulation Output}

To measure the results of optimization and simulation, numerous reports are generated and analyzed. The performance metrics (including inventory, costs, serviceability) are computed and recorded during the simulation. They are saved to the database and can be retrieved for further analysis. Summary tables are generated for the following performance metrics

- Serviceability: calculated as the percentage of orders that are shipped within the target, i.e. 7 days;

- Average order-to-ship time (days)
- Inventory and holding costs

- Transportation costs by mode (ocean or air)

\section{SIMULATION EXPERIMENTS AND RESULTS}

We study a number of business scenarios that are relevant to the business using GBSE and the simulation model described in the previous sections. Through simulation analysis we examine the impact of alternative supply chain strategies for each of the scenarios. The purpose of this simulation study is to analyze the factors that are affecting the performance of the supply chain and quantify the potential impact of the various initiatives that have been proposed for the supply chain.

We use the base model as described in the previous section to simulate the "as-is" scenario and use it to calibrate the model parameters used for the simulation model. This calibration process is needed to ensure the simulation model represents the current supply chain settings and the outputs produce realistic performance metrics. In the following subsections, we report some of the findings that we obtained through the simulation study. Each subsection focuses on a specific issue that is used as a key driver for the performance assessment.

\subsection{Impact of forecast accuracy}

Due to the long supply lead time, demand forecasts must be made several months in advance, and they are passed to the Tier 2 suppliers. It is a very challenging task to improve the forecast accuracy given that the lead time is so long. However, it is important to understand and quantify the impact of forecast accuracy so adequate efforts will be made to improve it.

For the business, forecasting is done at both the product level and the component level. While the product level forecast is given in terms of the volume per period, the component level forecast is provided in terms of feature ratios, which are the demand ratios between the components and the product that they are made into. Hence, we designed two sets of analyses: one for product level volume forecast accuracy and one for feature ratio forecast accuracy.

We simulated 2 sets of scenarios. The first set of scenarios is presented to show the impact of the product level forecast accuracy as it varies from $80 \%$ to $95 \%$ as shown in Figure 3. The impact of component level forecast accuracy is demonstrated by the second set of scenarios where the feature ratio forecast accuracy changes from $80 \%$ to $95 \%$ as shown in Figure 4 . From these results we can see that both transportation cost and inventory carrying cost decrease when forecast accuracy increases, and the serviceability performance in terms of the average order-to-ship lead time $(\mathrm{O} 2 \mathrm{~S})$ is also improved. If we com- 
pare the results from these two data sets, we can find that the changes in the first result appear to be more significant than the second one. This is because that any improvement in the forecast accuracy at the product level would directly reduce the mismatch between demand and supply. While at the component level, the improvement of the forecast accuracy is made to the forecasts of feature ratios which are typically fractional numbers. Therefore, the relative impact may seem to be less significant for the second set of scenarios with the same level of forecast accuracy improvement.

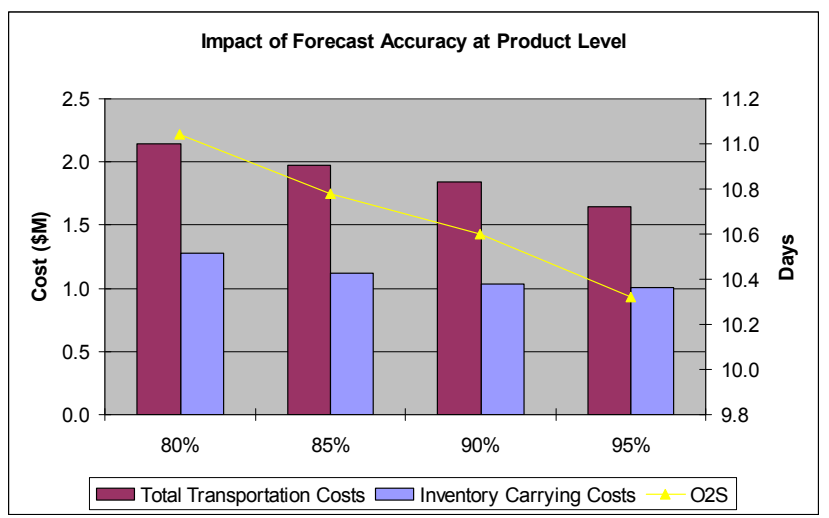

Figure 3: Impact of Forecast Accuracy at Product Level

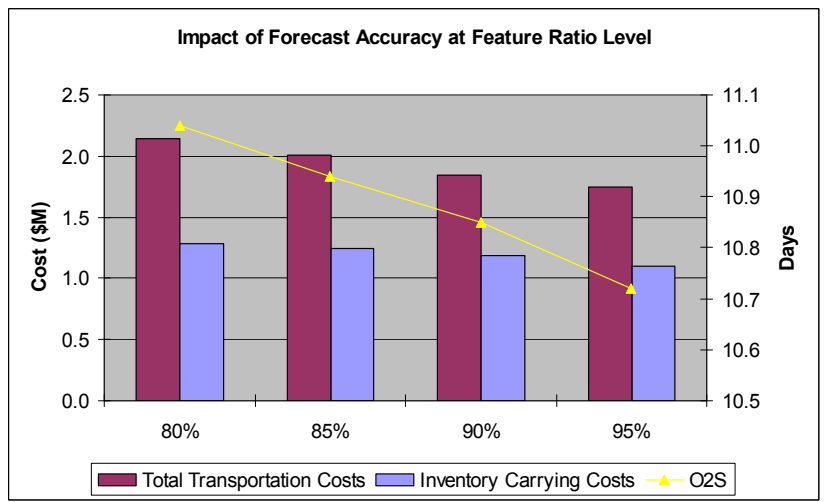

Figure 4: Impact of Forecast Accuracy at Feature Ratio Level

\subsection{Impact of BTO vs. BTP}

Given the long supply lead time, Tier 2 suppliers are typically required to build the components based on forecasted demands (or build plans). However, an exception is a particular component supplier who preferred build to order (BTO) for their products. Their rationale for using BTO was that the particular component is a high cost item and it needs to be built to customer's specification. Clearly such a practice was a root-cause for the poor customer servicea- bility for the related products since the supply lead time for the component is more than 2 weeks even with air transportation. Obviously switching from BTO to BTP is the only way to meet the serviceability target. However, a cost analysis is needed to find out what would be the cost impact for making such a change.

We simulated the scenarios of BTO and BTP for those particular components which are supplied by tier 2 suppliers. Figure 5 shows simulation results for the BTO and BTP with the cost and serviceability for the three tier 1 suppliers (Plant1, Plant2, and Plant3). It is found that as the process moves to BTP from BTO, service level is improved significantly as expected. For the cost part, transportation cost decreases and inventory carrying cost increases; while the total cost still decreases. Therefore, BTP seems to be a preferred process for tier 2 suppliers for the supply chain.

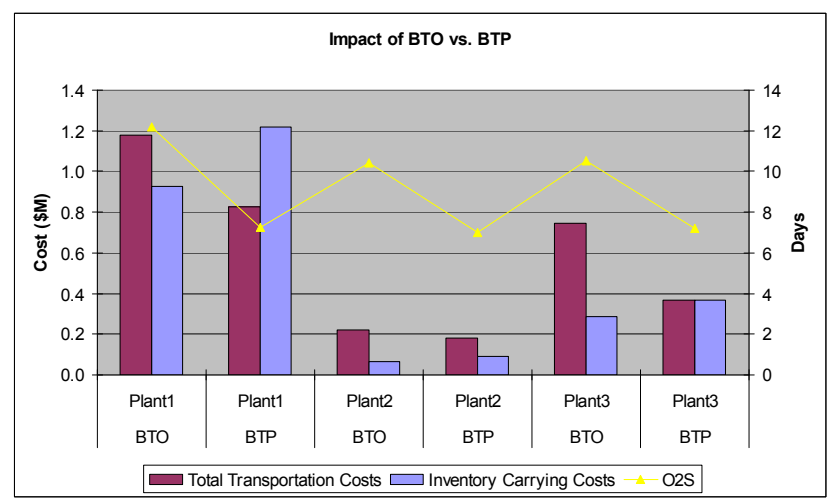

Figure 5: Impact of BTO vs. BTP

\subsection{Consolidated Product Scenario}

In order to deal with the long supply lead time and demand uncertainty associated with the customer orders, the business would like to explore a consolidated product process. The concept of this process is to build a few generic bare bone products to stock and fulfill the customer orders by customizing the bare bone products with a few additional components. The total number of bare bone products and components is much smaller; therefore, by taking advantage of the risk pooling effects, the total inventory to carry is substantially smaller. This is in fact the well-known "postponement" strategy which minimizes risk by delaying product customization until the last possible moment.

We simulated three scenarios with different degrees of product consolidations, and compared the results with the scenario of no consolidation (AS-IS) scenario. The number of product portfolio in the three consolidation scenarios are respectively $80 \%, 60 \%$ and $40 \%$ of the AS-IS product portfolio. The results show that both transportation cost and inventory carrying cost decrease considerably, and the servi- 
ceability is also improved significantly, as products are consolidated more.

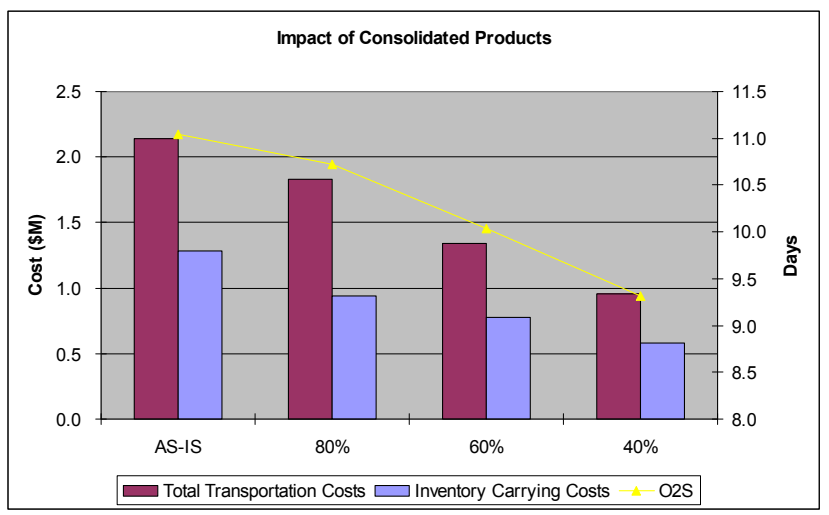

Figure 6: Impact of Consolidated Products

\subsection{Impact of the Premium Transportation Budget}

The use of premium shipments will guarantee to improve the service level significantly without the increase of inventory cost. However, the cost of premium shipments is substantially higher than that of regular shipments (by sea). The business manages the transportation costs by setting up a budget constraint, which limits the total cost that can be spent on premium shipments. The budget setting is an important business decision that impacts both the service level to customers and the profitability of the business.

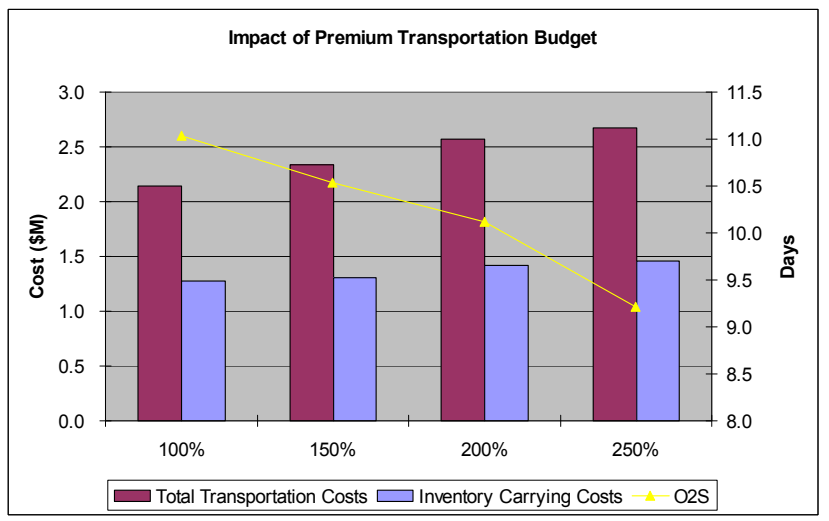

Figure 7: Impact of Premium Transportation Budget

We carried out an analysis to provide a quantitative view of the relationship between the premium ship budget and the supply chain performance in terms of serviceability and inventory costs. In this analysis, we assume that the premium transportation budget is split to weekly proportionally based on demand forecast. We study four scena- rios, and the total budgets for them are $100 \%, 150 \%, 200 \%$, and $250 \%$ of the original budget respectively. The results show that as the budget for premium transportation increases, the average order-to-ship lead time is reduced, and both transportation cost and inventory carrying cost are increased. This is because more premium shipping can be carried on to fulfill customer orders which could not be fulfilled in time in scenarios with lower budget. The simulation result is useful in making business decision for balancing transportation cost and serviceability.

\section{CONCLUDING REMARKS}

Efficient fulfillment strategy for the industrial solution supply chain requires careful understanding of interactions of many factors such as transportation modes, product configurations, forecast accuracy, and supply chain processes of multiple tiers of suppliers. Simulation is excellent way to understand the interactions and make intelligent business decision for balancing serviceability and costs. We describe simulation modeling capability that is used as a testbed for evaluating various ideas of improving effectiveness of the supply chain. We demonstrate the usefulness of the simulation tool by presenting sample simulation results. With the simulation tool, the business decision makers are better equipped in evaluating bold ideas before the costly implementation.

\section{REFERENCES}

Bagchi, S., S. Buckley, M. Ettl, and G. Lin, 1998. Experience Using the IBM Supply Chain Simulator, In Proceedings of the 1998 Winter Simulation Conference, ed. D.J. Medeiros, E.F. Watson, J.S. Carson and M.S. Manivannan.

Dong, L., H. Ding, C. Ren, and W. Wang, 2006. IBM SMARTSCOR - A SCOR Based Supply Chain Transformation Platform Through Simulation and Optimization Techniques, In Proceedings of the 2006 Winter Simulation Conference, ed. L.F. Perrone, F.P. Wieland, J. Liu, B.G. Lawson, D.M. Nicol, and R.M. Fujimoto.

Dietrich, B., D. Connors, T. Ervolina, J. Fasano, R. Lougee-Heimer, and R. Wittrock 2005. Applications of Implosion in Manufacturing. In: An, C. and Fromm, H. (eds.) Advances in Supply Chain Management, Springer.

Lin, G., R. Breitwieser, F. Cheng, J. Eagen, and M. Ettl, 2000. Product Hardware Complexity and Its Impact on Inventory and Customer On-Time Delivery, International Journal of Flexible Manufacturing Systems, 12(2-3):145-163. 
Scheller-Wolf, A., and S. Tayur. 1998. A Markovian DualSource Production-Inventory Model with Order Bands, GSIA Working Paper No. 1998-E200, Carnegie Mellon University.

Sethi, S. P., H. Yan, and H. Zhang. 2001. Peeling Layers of an Onion: Periodic Review Inventory Model with Multiple Delivery Modes and Forecast Updates, Journal of Optimization Theory and Applications, 108: 253-281.

Zhang, V.L. 1996. Ordering policies for an inventory system with three supply modes, Naval Research Logistics, 43(5): 691-708.

\section{AUTHOR BIOGRAPHIES}

FENG CHENG is a researcher in the Business Analytics and Mathematical Sciences Department at the IBM Thomas J. Watson Research Center, in Yorktown Heights, NY. He joined IBM Research Division in 1996 after receiving a $\mathrm{Ph}$.D. degree in Operations Research from University of Toronto. Dr. Cheng has worked on a wide range of research and business consulting projects in various areas including supply chain management, business process modeling, and risk management. He has published numerous research papers in scholarly journals and professional conferences of the related fields. His e-mail address is $<$ fcheng@us.ibm.com>.

YOUNG LEE is a Research Staff Member in the mathematical science department of IBM's T.J. Watson Research Center. Dr. Lee received B.S., M.S., and Ph.D. degrees in chemical engineering from Columbia University. He joined the IBM Research Division in 2002, and has been working in the areas of supply chain simulation and optimization. Prior to joining IBM, he had worked for BASF for 14 years, where he had founded and managed the Mathematical Modeling Group, and led development of numerous optimization and simulation models for various logistics and manufacturing processes. His research interest includes simulation and optimization of supply chain, manufacturing, services and business processes. His email address is <ymleedus.ibm. com>.

HONGWEI DING is a Researcher at IBM China Research Laboratory. He received his Ph.D. in Automation from INRIA (French National Institute of Computer Science \& Control), France. Before joined IBM, he was a researcher at INRIA. His research interests include supply chain modeling, optimization and simulation. His e-mail address is <dinghw@en. i.bm. com $>$.

WEI WANG is a Researcher at IBM China Research Laboratory. He joined IBM Research in 2005 after receiving his master degree in Control Science and Engineering from
Tsinghua University in Beijing, P. R. China. Currently, his research interests include supply chain simulation and optimization. His e-mail address is <wangwcrlecn.ibm.com>.

STUART STEPHENS is an Operations Manager in the IBM Integrated Supply Chain at Research Triangle Park, Raleigh, North Carolina. He Joined IBM Endicott in 1979 and has held numerous positions in Manufacturing, Materials Management, Product Planning, Integrated Supply Chain, and Operations Management. His e-mail address is <sbstephe@us.ibm.com>. 